VIII ${ }^{\text {èmes }}$ Journées Nationales Génie Civil - Génie Côtier, Compiègne, 7-9 septembre 2004

\title{
Efforts hydrodynamiques sur un cylindre vertical en zone côtière
}

\author{
Marilyne Luck, Jean-Michel Menon, Michel Benoît \\ Laboratoire National d'Hydraulique et Environnement (LNHE) \\ EDF R\&D 6 quai Watier - BP 49 - 78400 Chatou 2 France \\ Tel. :0130877666-e-mail : marilyne.luck@edf.fr
}

\section{Résumé}

Les efforts dus à la houle sur une structure de type monopile ont été étudiés par des essais en canal à houle sur un cylindre vertical, dans des conditions de faible profondeur d'eau jusqu'au déferlement. La comparaison des efforts mesurés aux prédictions obtenues (formule de Morison ${ }^{1}$ utilisant différentes méthodes de calcul de la cinématique de houle) montre la nécessité de disposer de méthodes précises pour la détermination de la cinématique de la houle en zone côtière. En effet, en faible profondeur d'eau, l'utilisation de la théorie linéaire conduit souvent à une sous-estimation des efforts ; 1'utilisation de méthodes basées sur la fonction de courant réduit cette sous-estimation, mais des travaux doivent encore être menés pour améliorer la prédiction des forces d'impact dues au déferlement.

\begin{abstract}
$\underline{\text { Abstract }}$
Wave-induced loadings acting on a monopile structure were investigated by experimental tests on a vertical cylinder in a wave flume with a sloping bottom, considering non-breaking, breaking and broken wave conditions. Measured forces were compared to numerical predictions obtained by applying Morison formula and by using different methods to compute the wave kinematics. This comparison highlights the need for accurate prediction methods for wave kinematics of shoaling and breaking waves. Indeed the linear wave theory often leads to significant underestimation of the measured forces. Using advanced stream function methods, this under-prediction is significantly reduced, but additional work is needed to better predict the impulsive force due to breaking.
\end{abstract}

Mots-clés: Eolien offshore, monopile, cinématique de houle, efforts hydrodynamiques, cylindre vertical, zone côtière.

\section{Contexte et objectifs:}

L'estimation des efforts dus à la houle sur des structures minces et par des profondeurs d'eau

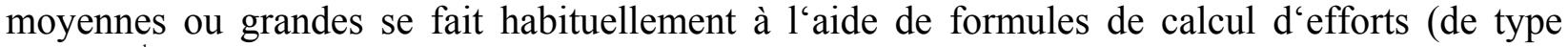
Morison ), à partir de la connaissance des conditions marines, locales ou 《 au large » de la structure. Cependant, pour les profondeurs moyennes ou faibles (inférieures à 20-25 m environ), la physique des processus côtiers $n$ 'est en général pas prise en compte par les méthodes et outils industriels (effets liés aux vagues très cambrées, dissymétriques, et surtout pouvant déferler). Ces spécificités liées aux faibles profondeurs d'eau rendent nécessaire le recours à des essais sur maquette pour déterminer les chargements. 
Depuis quelques années, des projets d'installation de parcs éoliens offshore en zone précôtière sont proposés. A ce titre, il est important d'améliorer la connaissance des efforts dus aux vagues sur les structures-supports d'éoliennes en faible profondeur d'eau, en particulier pour des structures de type « monopile».

Une étude expérimentale a été engagée au LNHE, avec la réalisation d'essais dans des conditions de faible profondeur d'eau et pour des vagues cambrées (du fait de la remontée des fonds) jusqu'au déferlement. L'objectif est double : (i) disposer de résultats expérimentaux pouvant être extrapolés à des conditions réelles, et (ii) valider et améliorer des outils et méthodes de prédiction de ces efforts.

\section{$\underline{\text { 2.Etude expérimentale en canal à houle }}$}

\subsection{Installation d'essais et structure étudiée}

Le modèle réduit est construit dans un canal à houle aléatoire du LNHE, de longueur utile 72 $\mathrm{m}$, de largeur $1.5 \mathrm{~m}$ et avec un niveau d'eau au repos pouvant aller jusqu'à $1.2 \mathrm{~m}$ devant le batteur. Ce canal est équipé d'un générateur de houle de type « piston », qui peut produire des vagues régulières ou irrégulières.

Les essais sont réalisés en similitude de Froude, à 1 'échelle $1 / 25^{\text {ime }}$. Les fonds sont représentés par un plan incliné de pente constante en ciment ; deux valeurs de pente ont été testées : 1/40= $2.5 \%$ et $1 / 20=5 \%$. Afin d'amortir la houle en bout de canal, un talus en enrochements a été mis en place (figure 1).

On considère une structure-support d'éolienne de type "monopile », représentée sur le modèle réduit par un cylindre vertical de diamètre $0.2 \mathrm{~m}$ (soit $5 \mathrm{~m}$ au réel), dont la base est positionnée $0.4 \mathrm{~m}$ au-dessus du fond « naturel » du canal.

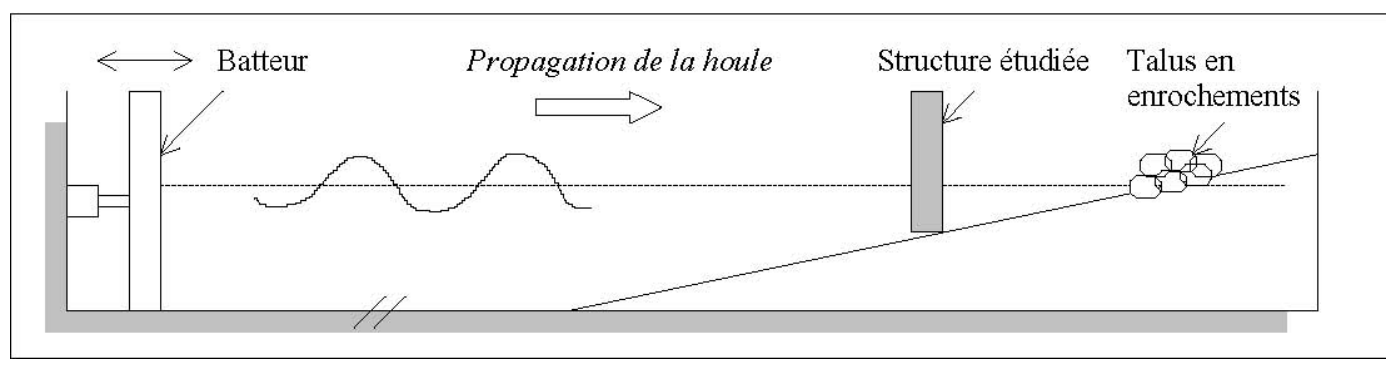

Figure 1 : Schéma de l'installation d'essais en canal à houle

\subsection{Mesures réalisées}

Des mesures de l'élévation de la surface libre sont réalisées au large et en différents points le long de la pente, par le biais de 11 capteurs résistifs, afin de déterminer les caractéristiques de la houle au large, de connaître les conditions de houle à l'approche et au niveau de la structure et de mesurer le run-up sur la structure (par un capteur situé directement à l'avant du cylindre).

Les efforts horizontaux sont mesurés par le biais de deux capteurs d'efforts, fonctionnant aussi bien en traction qu'en compression et entièrement étanches. Ces capteurs sont positionnés à $0.2 \mathrm{~m}$ et $0.8 \mathrm{~m}$ du fond et constituent les uniques liaisons entre le cylindre et une poutre verticale servant de bâti fixe (figure 2).

L'acquisition des signaux de surface libre et d'efforts est réglée de façon à enregistrer au moins 50 vagues et à obtenir de 1'ordre de 80 à 100 points par vague. 


\subsection{Conditions d'essais}

Les essais ont été réalisés sans courant et en houle régulière. Différentes périodes et hauteurs de houle ont été simulées au large, de façon à couvrir une large gamme de conditions de houle au niveau de la structure (vagues déferlantes ou non).

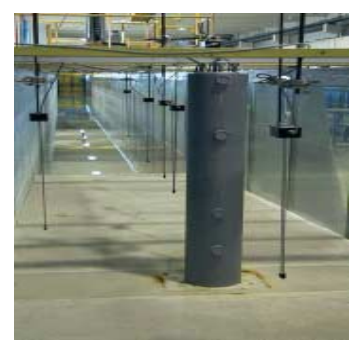

figure 2: Cylindre vu de l'arrière

(on distingue lebatteur à houle à l'extrémité du canal, en arrière plan)

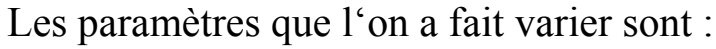

- les paramètres de houle au large (période $\mathrm{T}$ et hauteur incidente $\mathrm{H}_{0}$ )

Les périodes de houle testées varient de1.6 à $2.4 \mathrm{~s}$ sur modèle réduit ( 8 à $12 \mathrm{~s}$ au réel). Les hauteurs incidentes $\mathrm{H}_{0}$ sont choisies de manière à couvrir des conditions allant des vagues modérées jusqu'aux vagues déferlantes

-la profondeur d'implantation de la structure (d) et la pente des fonds (m)

Deux pentes ont été testées $(\mathrm{m}=2.5$ et $5 \%)$, ainsi que 4 profondeurs d'eau en pied de structure : $0.30,0.40,0.60$ et $0.80 \mathrm{~m}$ (soit $7.5,10,15$ et $20 \mathrm{~m}$ au réel).

$\mathrm{Au}$ total, 128 essais ont ainsi été réalisés en houle régulière, chacun correspondant à une valeur du quadruplet $\left(T, H_{0}, m, d\right)$.
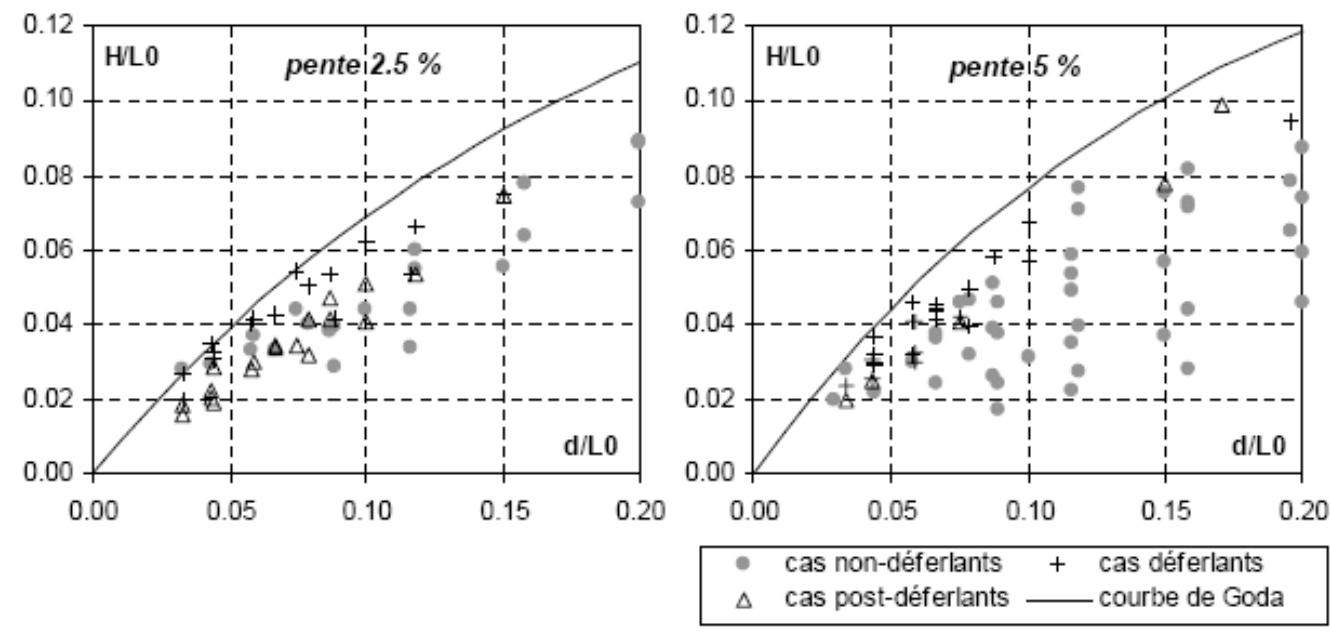

Figure 3 : Caractéristiques des conditions de houle pour les essais réalisés

Les caractéristiques de ces essais sont synthétisés sur la figure 3, présentant sur un diagramme adimensionnel $\mathrm{H} / \mathrm{L}_{0}=\mathrm{f}(\mathrm{d} / \mathrm{L} 0)$, où $\mathrm{H}$ est la hauteur de houle au niveau

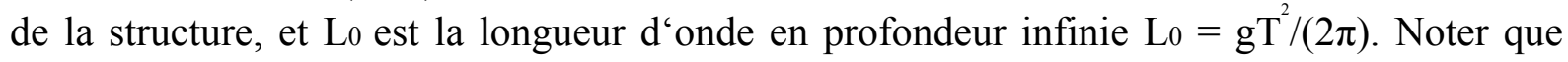
sont utilisés des symboles différents pour distinguer les cas non-déferlants (la houle déferle après 
le cylindre), déferlants (la houle déferle sur ou au voisinage du cylindre), ou post-déferlants (la houle déferle avant le cylindre). La figure 3 montre que les conditions d'essais couvrent une large gamme de valeurs de $\mathrm{d} / \mathrm{L} 0$ (de 0.03 à 0.2 ) et $\mathrm{H} / \mathrm{L}_{0}$ (de 0.015 à 0.1 ), avec de nombreux points au voisinage de la courbe correspondant au critère de déferlement de Goda ${ }^{2}$.

\section{Extrema des efforts horizontaux et moments de renversement mesurés}

Les signaux mesurés (élévations de surface libre et efforts horizontaux) ont été moyennés sur une série de cinq vagues successives, afin d'obtenir des profils temporels caractéristiques sur une période de houle. Un exemple de signaux mesurés est donné sur la figure 4 pour un essai particulier.
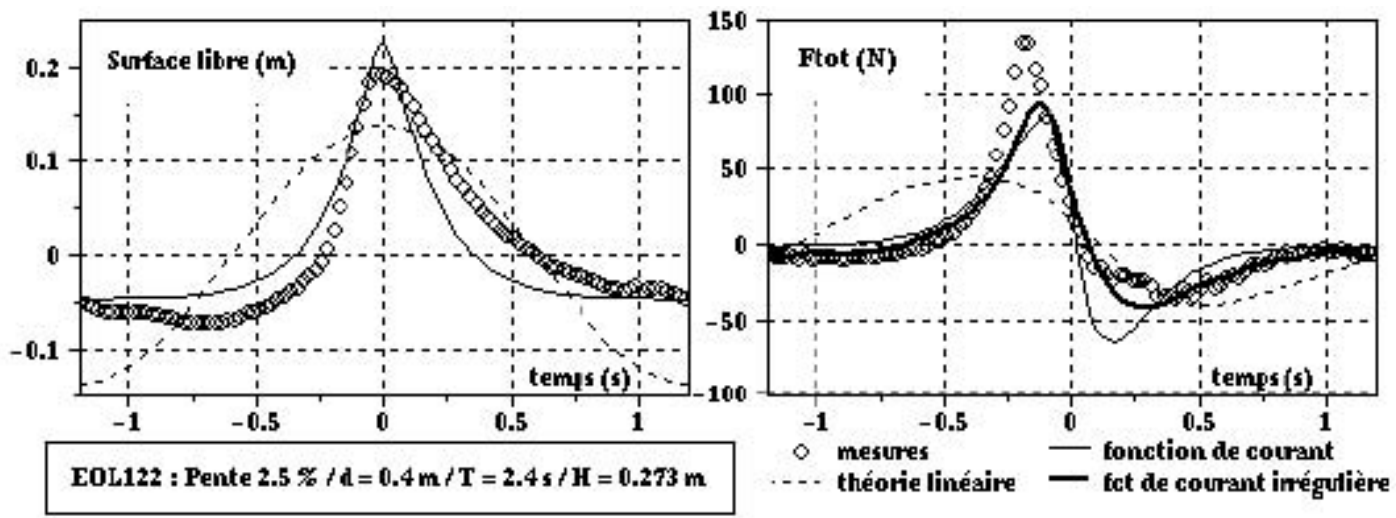

Figure 4 : Exemple de profils temporels de surface libre et d'effort horizontal pour un essai particulier, avec comparaison à des modèles de prédiction

Les valeurs maximales et minimales (sur une période de houle) de l'effort horizontal total et du moment de renversement total mesurés sont représentées sur la figure 5, en fonction de la hauteur de houle locale (i.e. au niveau du cylindre).

Les efforts maximaux mesurés (efforts positifs, dans le sens de propagation de la houle) s'étalent d'environ 25 à $265 \mathrm{~N}$ (soit 390 à $4140 \mathrm{kN}$ au réel), et les efforts minimaux (efforts négatifs, dans le sens opposé à celui de propagation de la houle) varient entre 15 et $110 \mathrm{~N}$ (soit 235 à $1718 \mathrm{kN}$ au réel). Ces efforts et moments maximaux sont en général bien corrélés à la hauteur de houle locale et à la profondeur en pied de cylindre : ils augmentent globalement avec

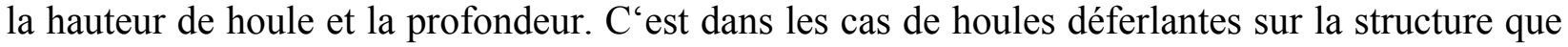
les efforts et moments sont les plus importants (effort d'impact des vagues déferlantes). La figure 5 indique que, pour une profondeur fixée, on peut avoir une amplification d'un facteur de 2 à 3 dans le cas d'une houle déferlante, par rapport à une houle non-déferlante de même hauteur locale (mais de période différente).

En comparant les résultats pour les profondeurs 0.4 et $0.8 \mathrm{~m}$ (soit 10 et $20 \mathrm{~m}$ au réel), on note aussi qu'une même hauteur de houle locale peut induire des efforts plus importants dans le cas de la profondeur $0.4 \mathrm{~m}$, si elle est déferlante. Par contre, le déferlement $\mathrm{n}^{\text {'a }}$ pas d'effet significatif sur les efforts minimaux. 

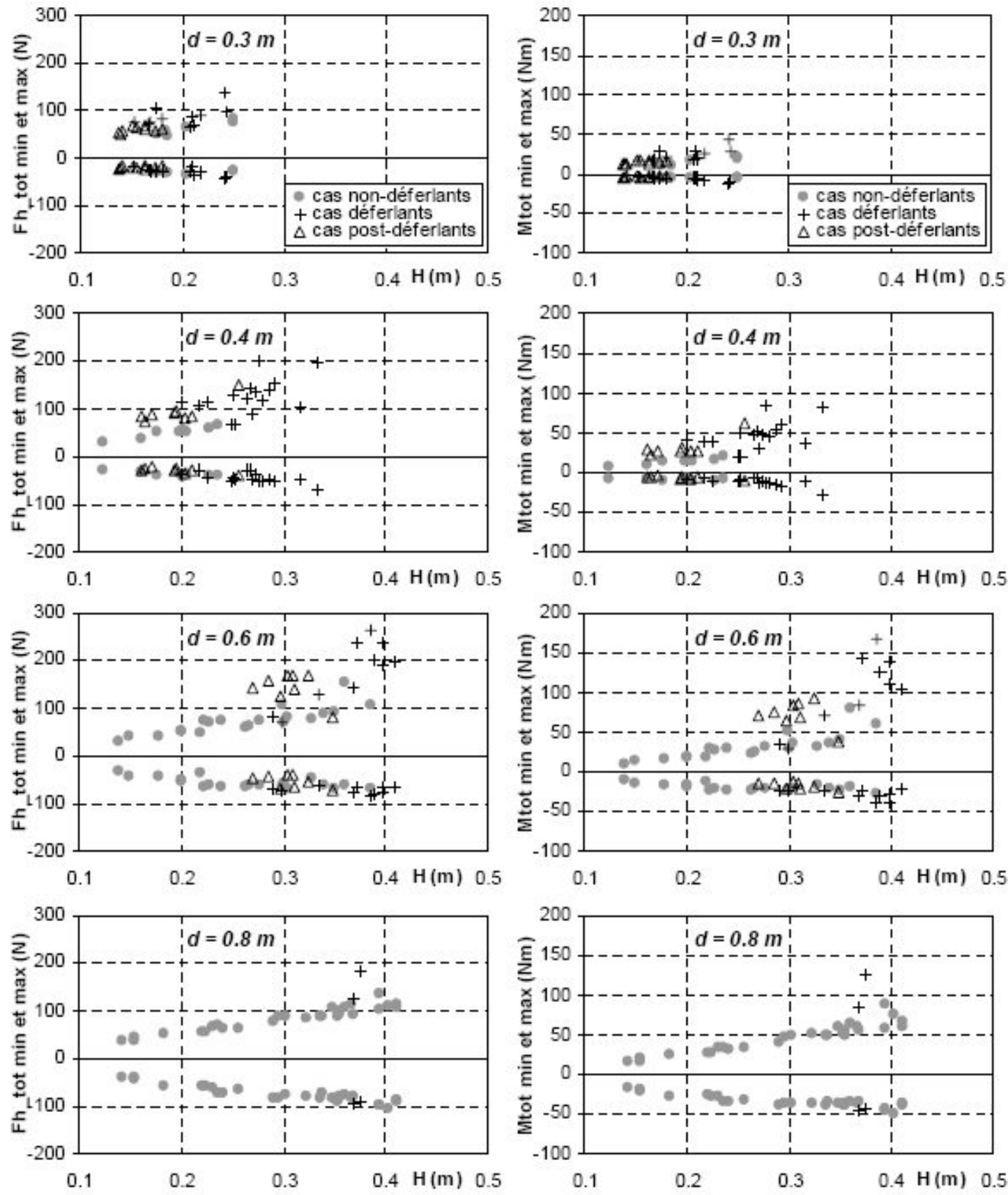

Figure 5 : Extrema de l'effort horizontal et du moment de renversement mesurés 


\section{Comparaison des résultats d'essais à des modèles de prédiction d'efforts}

\subsection{Approches de modélisation retenues pour l'évaluation des efforts}

La prédiction des efforts est basée sur l'utilisation de la formule dite de Morison1 pour une structure cylindrique de diamètre $\mathrm{D}$ faible devant la longueur d'onde L. Cette approche est pertinente vu que nos essais présentent des rapports $\mathrm{D} / \mathrm{L}$ toujours inférieurs à 0.1 . La force horizontale théorique s'exerçant sur un élément de cylindre vertical de hauteur dz, situé à la cote z est donnée par l'expression :

$$
\mathrm{dF}^{\mathrm{h}}(\mathrm{z}, \mathrm{t})=\mathrm{dF}_{\text {trânée }}^{\mathrm{h}}+\mathrm{dF}_{\text {inertie }}^{\mathrm{h}}=\frac{1}{2} \rho \mathrm{C}_{\mathrm{D}} \mathrm{D}|\mathrm{u}(\mathrm{z}, \mathrm{t})| \mathrm{u}(\mathrm{z}, \mathrm{t}) \mathrm{dz}+\rho \mathrm{C}_{\mathrm{M}} \frac{\pi \mathrm{D}^{2}}{4} \mathrm{a}(\mathrm{z}, \mathrm{t}) \mathrm{dz}
$$

Cette force est fonction de la cinématique de la houle (vitesse horizontale $u(z, t)$ et accélération horizontale $a(z, t)$ calculées en l'absence de structure) et des coefficients hydrodynamiques de traînée CD et d'inertie CM du cylindre, dépendant des caractéristiques de l'écoulement et de la structure étudiée (valeurs caractéristiques se trouvant entre 0.5 et 1.4 pour $\mathrm{CD}$ et entre 1.5 et 2 pour $\mathrm{CM}$ ). La force horizontale totale est obtenue par intégration sur la hauteur immergée du cylindre entre le fond $(z=-d)$ et la surface libre $(z=\eta(t))$ :

$$
\mathrm{F}_{\text {totale }}^{\mathrm{h}}(\mathrm{t})=\int_{-\mathrm{d}}^{\eta(\mathrm{t})}\left[\frac{1}{2} \rho \mathrm{C}_{\mathrm{D}} \mathrm{D}|\mathrm{u}(\mathrm{z}, \mathrm{t})| \mathrm{u}(\mathrm{z}, \mathrm{t})+\rho \mathrm{C}_{\mathrm{M}} \frac{\pi \mathrm{D}^{2}}{4} \mathrm{a}(\mathrm{z}, \mathrm{t})\right] \mathrm{dz}
$$

Le moment de renversement total s'obtient de façon analogue :

$$
\mathrm{M}_{\text {total }}^{\mathrm{r}}(\mathrm{t})=\int_{-\mathrm{d}}^{\eta(\mathrm{t})}\left[\frac{1}{2} \rho \mathrm{C}_{\mathrm{D}} \mathrm{D}|\mathrm{u}(\mathrm{z}, \mathrm{t})| \mathrm{u}(\mathrm{z}, \mathrm{t})+\rho \mathrm{C}_{\mathrm{M}} \frac{\pi \mathrm{D}^{2}}{4} \mathrm{a}(\mathrm{z}, \mathrm{t})\right](\mathrm{d}+\mathrm{z}) \mathrm{dz}
$$

Pour la détermination de la cinématique de la houle, nous avons mis en oeuvre et comparer trois méthodes de calcul :

- la théorie de la houle linéaire (Airy3), classiquement utilisée, mais peu adaptée pour des vagues très cambrées, car fortement non linéaires ;

- la méthode de fonction de courant « classique » (Dean4), permettant, à partir de la hauteur des vagues et de leur période, un calcul « exact» pour des vagues stables se propageant sans déformation sur un fond plat. Cette approche modélise l'asymétrie des vagues par rapport à un plan horizontal ;

- la méthode de fonction de courant « irrégulière » (Dean4, Chakrabarti et al.5). Par rapport à la méthode précédente qui se base uniquement sur la hauteur et la période, celle-ci utilise en condition d'entrée le profil de surface libre mesuré sur une période, ce qui permet de représenter l'asymétrie des vagues par rapport à un plan horizontal et à un plan vertical. Les caractéristiques de la houle utilisées en données d'entrée pour ces différentes théories sont les conditions de houle locales au niveau de la structure, mesurées dans la même section transversale du canal que la structure. Pour les coefficients hydrodynamiques, nous avons utilisé dans une première analyse des valeurs indiquées par Sarpkaya et Isaacson6, à savoir $\mathrm{CD}=0.7$ et $\mathrm{CM}=1.8$ (cas d'un cylindre lisse dans un écoulement à nombre de Reynolds élevé). 


\subsection{Analyse des performances des méthodes de prédiction d'efforts}

\subsubsection{Modélisation des profils temporels d'efforts horizontaux}

Un exemple de comparaison des profils temporels mesurés et prédits de l'effort horizontal total est donné sur la figure 4 pour un cas de vague déferlante. On voit que la théorie linéaire ne permet pas de bien représenter l'effort mesuré, tandis que les méthodes de fonction de courant améliorent nettement les prédictions, notamment la méthode fonction de courant irrégulière. Des figures analogues à la figure 4 permettent une évaluation visuelle de la validité de nos prédictions, mais n'offrent pas une mesure quantitative de cette validité. De plus, compte-tenu du nombre d'essais réalisés, il est difficile de présenter tous les graphiques pouvant ainsi être construits. Afin d'obtenir des indicateurs quantitatifs de validité des méthodes de prédiction des profils d'efforts horizontaux, nous avons donc utilisé un indicateur d'erreur $\delta$, proposé par Sumer et Fredsøe7, et défini par:

$\delta=\left[\int_{t=0}^{\mathrm{T}}\left[\mathrm{F}_{\text {mes }}(\mathrm{t})-\mathrm{F}_{\text {calc }}(\mathrm{t})\right]^{2} \mathrm{dt}\right] /\left[\int_{\mathrm{t}=0}^{\mathrm{T}}\left[\mathrm{F}_{\text {mes }}(\mathrm{t})\right]^{2} \mathrm{dt}\right]$

où Fmes et Fcalc sont les efforts mesurés et prédits, et $\mathrm{T}$ la période de la houle.

Ce paramètre $\delta$ représente l'erreur moyenne entre les deux séries temporelles d'efforts. Les valeurs du paramètre $\delta$ obtenues avec les trois méthodes de prédiction apparaissent sur la figure 6 , en fonction du ratio $\mathrm{H} / \mathrm{d}$ local.
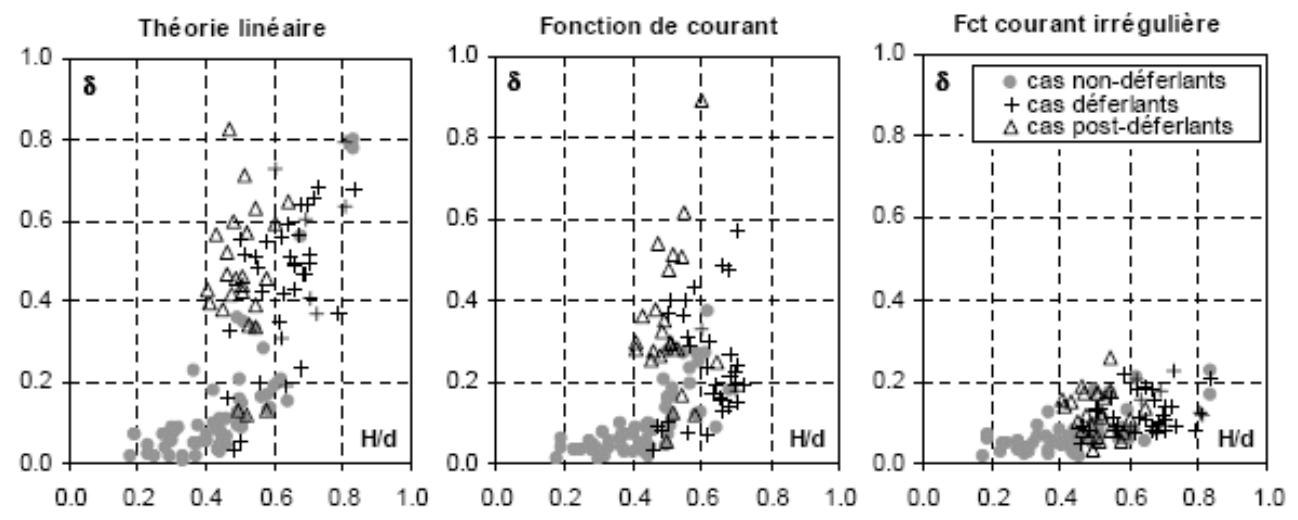

Figure 6 : Evolution du paramètre d'erreur $\delta$ entre l'effort total mesuré et calculé (pour les trois théories de houle utilisées) en fonction de $\mathrm{H} / \mathrm{d}$

On note qu'avec la théorie linéaire, même dans les cas sans déferlement, le paramètre $\delta$ prend des valeurs élevées (supérieures à 0.2 , et souvent supérieures à 0.4 pour les cas déferlants ou post-déferlants). L'utilisation de la méthode de fonction de courant classique permet d'obtenir des valeurs de $\delta$ inférieures, à savoir toujours comprises entre 0 et 0.4 dans le cas non déferlants, mais pouvant tout de même atteindre 0.9 dans le cas de vagues post-déferlantes, présentant un profil temporel très asymétrique en forme de dent de scie.

En outre, il faut signaler que cette méthode ne fonctionne que si le rapport $\mathrm{H} / \mathrm{d}$ est inférieur à la limite théorique de 0.78 . 
Or cette valeur est dépassée très souvent sur nos essais pour les cas déferlants, et donc la méthode ne donne pas de résultats sur ces cas (comme on peut le voir en comparant le graphe du milieu de la figure 6 aux deux autres : il y a nettement moins de points pour les valeurs de H/d supérieures à 0.7 ).

La méthode de fonction de courant irrégulière fonctionne quant à elle sur tous les cas considérés et conduit à des valeurs de $\delta$ nettement améliorées, quasiment toujours inférieures à 0.2 , excepté pour certains cas déferlants ou post-déferlants.

\subsubsection{Prédiction des efforts extrêmes}

Dans un second temps, les extrema (positifs et négatifs) des efforts horizontaux mesurés et prédits ont été comparés et les résultats sont présentés sur la figure 7.
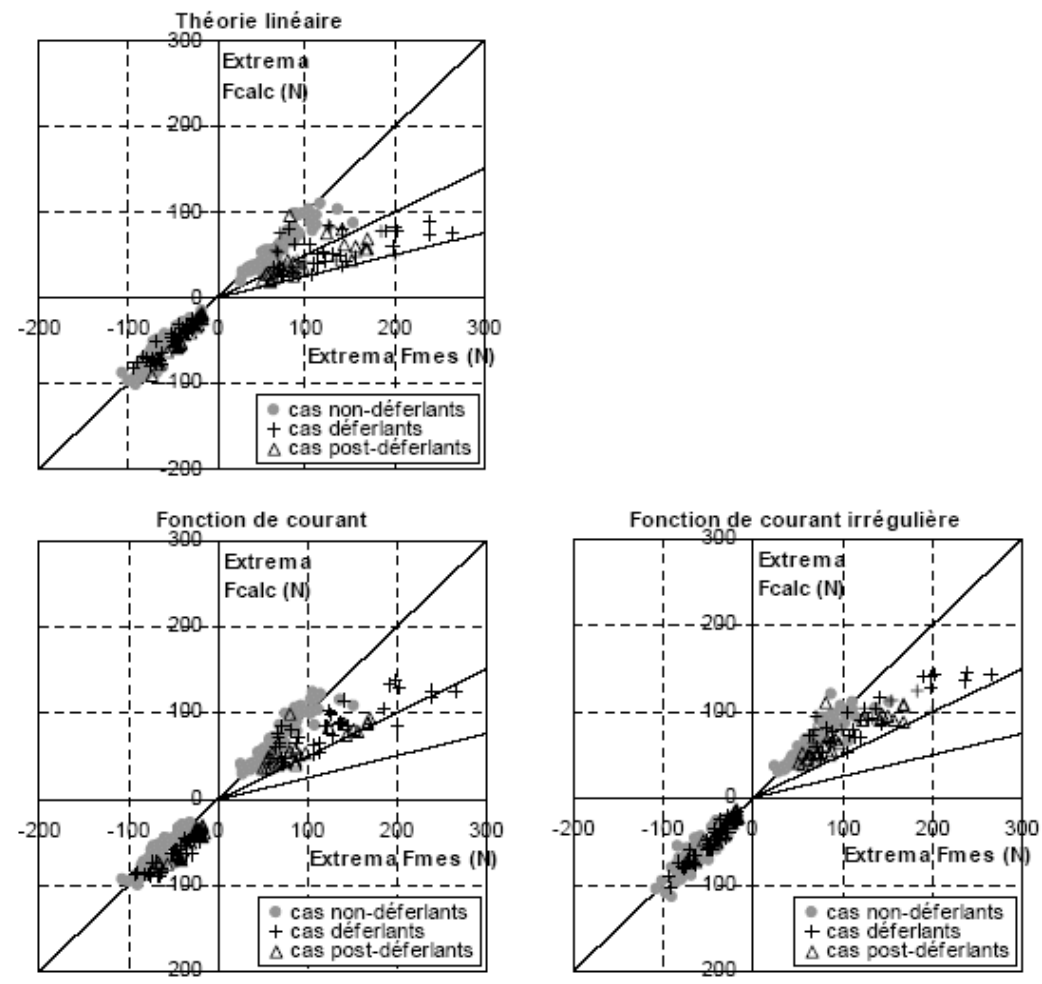

Figure 7 : Comparaison des extrema d'efforts mesurés et calculés avec les différentes théories pour le calcul de la cinématique de houle

Sur les trois graphes de cette figure (un par méthode de calcul de la cinématique de la houle), les droites correspondant à une bonne prédiction (droite $\mathrm{y}=\mathrm{x}$ ), ainsi qu'à une sous-estimation d'un facteur 2 et d'un facteur 4 des extrema des efforts mesurés ont également été tracées.

En ce qui concerne les efforts minimaux (sens opposé au sens de propagation de la houle), les trois méthodes donnent des prédictions de bonne qualité, pour toutes les conditions de houle. La méthode de fonction de courant irrégulière minimise la dispersion du nuage des points autour la 
droite correspondant à une prédiction exacte, mais les prédictions de la théorie linéaire sont à peine moins bonnes.

En ce qui concerne les efforts maximaux (dans le sens de propagation de la houle), pour les cas non-déferlants au niveau de la structure, les estimations des maxima données par les différentes théories sont proches des maxima mesurés, avec des rapports Fmes_max/Fcalc_max en général compris entre 0.8 et 1.3 lorsqu'on met en œuvre les théories de la fonction de courant, et allant de 1 à 1.5 (voire 2) pour la théorie linéaire. Dans les cas déferlants ou post-déferlants, les prédictions sont moins bonnes : le ratio entre effort maximum mesuré et calculé peut atteindre 2.4 pour la théorie de la fonction de courant classique et 4 pour la théorie linéaire.

Pour la méthode de fonction de courant irrégulière, les estimations sont un peu meilleures, mais le ratio est compris entre 0.75 et 2 (i.e. la sous-estimation peut encore être d'un facteur 2). On peut noter que l'utilisation des méthodes de fonction de courant conduit parfois à surestimer les forces, alors que la théorie linéaire les sous-estime toujours (ratio Fmes_max/Fcalc_max toujours supérieur à 1).

\section{$\underline{\text { 5.Conclusions et perspectives de recherche }}$}

Les résultats obtenus à ce stade de l'étude montrent que des estimations acceptables de l'effort horizontal total dû à la houle peuvent être obtenues dans la zone de levée (ou " shoaling ») par application de la formule de Morison, avec une cinématique de houle calculée par une méthode de fonction de courant irrégulière. Cependant, dans le cas de vagues déferlantes, cette méthode sousestime l'effort subi par la structure : les forces peuvent être jusqu'à 2 fois supérieures aux prédictions, du fait du terme d'impact de déferlement. En comparaison, lorsqu'on utilise la théorie linéaire de houle, les efforts maximaux peuvent être sous-estimés d'un facteur 4. La méthode de fonction de courant classique améliore les prédictions de la théorie linéaire, mais son intérêt est limité pour les vagues déferlantes, vu qu'elle ne fonctionne que si $\mathrm{H} / \mathrm{d}<0.78$.

La comparaison des différentes théories de houle montre que la prise en compte de l'asymétrie des vagues par rapport à un plan vertical (théorie de la fonction de courant irrégulière) conduit à des prédictions meilleures que celles obtenues avec des théories de houle supposant un profil asymétrique uniquement par rapport à un plan horizontal (théorie de la fonction de courant classique). L'analyse des essais met ainsi en évidence la nécessité de disposer de méthodes décrivant correctement l'hydrodynamique des vagues cambrées et déferlantes.

Des travaux complémentaires sur la recherche de valeurs adaptées des coefficients hydrodynamiques (déterminées par application d'une méthode des moindres carrés entre mesures et prédictions) et sur la modélisation de la composante d'impact due au déferlement doivent encore être réalisés, afin d'améliorer l'estimation des efforts pour les vagues déferlant sur la structure.

\section{Références}

1 Morison J.R., O’Brien M.P., Johnson J.W., Schaaf S.A., 1950. The force exerted by surface waves on piles. Petroleum Transactions, AIME, Vol. 189, TP 2846, pp 149-154.

2 Goda Y., 1970. A synthesis of breaker indices. Trans. Japan Society of Civil Eng., Vol. 2, pp 227-230.

3 Airy G.B., 1845. Tides and waves. Encyclopaedia Metropolitana, Art. 192, pp 241-369. 
4 Dean R.G., 1965. Stream-function representation of non-linear ocean waves. J. Geophys. Res., Vol. 70, n¹8, pp 4561-4572.

5 Chakrabarti S.K., Kriebel D., Berek E.P., 1997. Force on a single pile caisson in breaking wave and current. Applied Ocean Research, Vol. 19, pp 113-140.

6 Sarpkaya T., Isaacson M., 1981. Mechanics of wave forces on offshore structures. Van Nostrand Reinhold Company Inc., 650 p.

7 Sumer B.M., Fredsøe J., 1997. Hydrodynamics around cylindrical structures. Advanced Series on Ocean Engineering, Vol. 12, World Scientific, 530 p. 